\section{First Integrals and Solutions of Duffing-van der Pol Type Equations}

\author{
Firdaus E. Udwadia \\ Professor \\ Departments of Aerospace and Mechanical Engineering, \\ Civil Engineering, Mathematics, \\ Systems Architecture Engineering, \\ and Information and Operations Management, \\ University of Southern California, \\ 430K Olin Hall, \\ Los Angeles, CA 90089 \\ e-mail: fudwadia@usc.edu
}

\author{
Hancheol Cho \\ Department of Aerospace and Mechanical Engineering, \\ University of Southern California, \\ Los Angeles, CA 90089 \\ e-mail: hancheoc@usc.edu
}

A simple transformation is used to obtain the first integrals and the solutions of the Duffing-van der Pol type equation under certain conditions. It is shown that the system can be totally integrable and this total integrability admits new solutions. The new solutions require weaker conditions on the system's parameters than hereto known. [DOI: 10.1115/1.4024673]

\section{Introduction}

Many dynamic models of physical systems are represented by nonlinear differential equations. Among them, the Duffing-van der Pol type equation has attracted numerous researchers due to its usefulness in modeling various physical systems. However, the nonlinearity of the equation makes it very difficult to find the first integrals and/or its solutions. Finding the first integrals of the motion is intricately connected with the inverse problem of the calculus of variations [1]. The standard method to find the first integrals is first to obtain a Lagrangian function from the differential equations describing the given mechanical system [2-4] and then to calculate the Jacobi integral [5] if the Lagrangian function does not contain time explicitly. In Ref. [4], for example, the first integrals of some linearly damped multidegree-of-freedom systems are reported. Prelle and Singer [6] proposed another procedure to find the first integrals of first-order ordinary differential equations of the form $\dot{x}=P(t, x) / Q(t, x)$ where both $P(t, x)$ and $Q(t, x)$ are polynomials. Duarte et al. [7] extended this method to second-order ordinary differential equations. In addition, Lie symmetry methods [8] have received considerable attention for their generality. Recently, the extended Prelle-Singer method and the Lie symmetry method have been applied to the Duffing-van der Pol type equation to obtain the first integrals and its solutions in some very special situations $[9,10]$. In Refs. $[9,10]$, a new first integral and a solution in a parametric form are reported under certain conditions. The purpose of this paper is to obtain the same first integral of the Duffing-van der Pol type equation for a much wider set of conditions by using a simpler and more direct approach without recourse to extensive symbolic computations and Lie symmetry methods. Moreover, implicit and explicit

Manuscript received December 14, 2012; final manuscript received May 18, 2013; accepted manuscript posted May 29, 2013; published online September 18, 2013. Assoc. Editor: Wei-Chau Xie. solutions for more general cases, which have not yet been obtained so far, are developed.

\section{Integrability and First Integrals of the Duffing-van der Pol Type Equation}

In this section, we derive the first integral of the Duffing-van der Pol type equation using a simpler, more direct method and obtain a more general solution, compared with the ones proposed in Refs. $[9,10]$ and so far known. The main idea is to use a suitable transformation and then obtain conditions under which the transformed equation is totally integrable. We begin with the following form of the Duffing-van der Pol type system:

$$
\ddot{x}+\beta\left(x^{2}-\lambda\right) \dot{x}+\gamma x+\alpha x^{3}=0
$$

where $x(t)$ is a generalized displacement, dots denote differentiation with respect to time $t$, and $\beta, \lambda, \gamma$, and $\alpha$ are constants. Furthermore, the case $\beta=0$ is excluded, since otherwise Eq. (2.1) does not have any damping term, yielding the first integral and the solution very easily. Using the transformation

$$
t=\nu \ln \tau
$$

where $\nu$ is a nonzero constant, Eq. (2.1) becomes

$$
\frac{d^{2} x}{d \tau^{2}}=-\beta \nu \tau^{-1} x^{2} \frac{d x}{d \tau}+(\beta \lambda \nu-1) \tau^{-1} \frac{d x}{d \tau}-\gamma \nu^{2} \tau^{-2} x-\alpha \nu^{2} \tau^{-2} x^{3}
$$

When $v$ is positive, the transformation in Eq. (2.2) corresponds to an expansion in the time scale. Now we show that by using only the single transformation Eq. (2.2), the first integrals of Eq. (2.1) can be directly obtained for a wide class of problems. Furthermore, implicit and explicit solutions are also possible under some conditions.

Consider the function

$$
G(\tau, x(\tau))=G_{1} \tau^{-1} x+G_{2} \tau^{-1} x^{3}
$$

where $G_{1}$ and $G_{2}$ are constant. The first derivative of Eq. (2.4) with respect to $\tau$ yields

$$
\frac{d G}{d \tau}=-G_{1} \tau^{-2} x+G_{1} \tau^{-1} \frac{d x}{d \tau}-G_{2} \tau^{-2} x^{3}+3 G_{2} \tau^{-1} x^{2} \frac{d x}{d \tau}
$$

Comparing Eq. (2.5) with the right hand side of Eq. (2.3), we have

$$
G_{1}=\gamma \nu^{2}, G_{1}=\beta \lambda \nu-1, G_{2}=\alpha \nu^{2}, 3 G_{2}=-\beta \nu
$$

or

$$
\gamma=\frac{\beta \lambda \nu-1}{\nu^{2}}, \quad \alpha=-\frac{\beta}{3 \nu}
$$

Eliminating $\nu$ from these relations yields

$$
\gamma=-\frac{9 \alpha^{2}}{\beta^{2}}\left(1+\frac{\beta^{2} \lambda}{3 \alpha}\right)
$$

so that, Eq. (2.1) becomes

$$
\ddot{x}+\beta\left(x^{2}-\lambda\right) \dot{x}-\frac{9 \alpha^{2}}{\beta^{2}}\left(1+\frac{\beta^{2} \lambda}{3 \alpha}\right) x+\alpha x^{3}=0
$$

where $\alpha \neq 0$ is assumed, and considering Eq. (2.7), $\beta \neq 0$. Combining Eqs. (2.3) and (2.5) then yields

$$
\frac{d^{2} x}{d \tau^{2}}=\frac{d G}{d \tau}
$$


under the constraints given by Eq. (2.7) or Eq. (2.8). Integrating Eq. (2.10) with respect to $\tau$ once gives

$$
\frac{d x}{d \tau}=G+C_{0}=G_{1} \tau^{-1} x+G_{2} \tau^{-1} x^{3}+C_{0}
$$

where $C_{0}$ is an integration constant, and $G_{1}$ and $G_{2}$ are determined by Eq. (2.6). Using Eq. (2.2), Eq. (2.11) becomes

$$
\nu \dot{x}=G_{1} x+G_{2} x^{3}+C_{0} e^{\frac{t}{\nu}}
$$

or

$$
\left(\dot{x}-\frac{G_{1}}{\nu} x-\frac{G_{2}}{\nu} x^{3}\right) e^{-\frac{t}{\nu}}=\frac{C_{0}}{\nu}:=I
$$

Using Eqs. (2.2), (2.6), and (2.8), Eq. (2.13) can be rewritten as

$$
\left(\dot{x}-\frac{3 \alpha+\beta^{2} \lambda}{\beta} x+\frac{\beta}{3} x^{3}\right) e^{\frac{3 \alpha}{\beta} t}=I
$$

which is a first integral of Eq. (2.1) when the relation given in Eq. (2.8) is satisfied, i.e., we have the first integral of Eq. (2.9) for any set of parameters $\alpha, \beta$, and $\lambda$. When $\alpha=1$ as in Refs. $[9,10]$, Eq. (2.14) becomes

$$
\left(\dot{x}-\left(\beta \lambda+\frac{3}{\beta}\right) x+\frac{\beta}{3} x^{3}\right) e^{\frac{3}{\beta^{t}}}=I
$$

which is the same as the first integral obtained in Refs. $[9,10]$. It must be noted that unlike in Refs. [9,10], the first integral Eq. (2.15) is derived using only one transformation, Eq. (2.2), and without the use of extensive symbolic computations. Instead, total integrability of the transformed equation Eq. (2.3) is focused upon, and this insight helps in finding the first integral directly.

\section{New Solutions of the Duffing-van der Pol Type Equation}

Since the first integral, Eq. (2.14), is obtained, by integrating this first-order differential equation one can get a solution to the Duffing-van der Pol type system given by Eq. (2.9). The simplest case occurs when $I=0$. At the initial time $t=0$, Eq. (2.14) becomes

$$
\dot{x}_{0}-\frac{3 \alpha+\beta^{2} \lambda}{\beta} x_{0}+\frac{\beta}{3} x_{0}^{3}=I
$$

where $x_{0}$ and $\dot{x}_{0}$ are the initial displacement and velocity, respectively. If we choose the initial conditions $x_{0}$ and $\dot{x}_{0}$ so that $I=0$, that is,

$$
\dot{x}_{0}=\frac{3 \alpha+\beta^{2} \lambda}{\beta} x_{0}-\frac{\beta}{3} x_{0}^{3}
$$

then Eq. (2.14) becomes totally integrable

$$
\frac{d x}{d t}=\frac{3 \alpha+\beta^{2} \lambda}{\beta} x-\frac{\beta}{3} x^{3}
$$

The solution to the first-order differential equation Eq. (3.3) is easily obtained

$x(t)=\frac{ \pm 1}{\sqrt{\frac{\beta^{2}}{3\left(3 \alpha+\beta^{2} \lambda\right)}-\Gamma e^{-\frac{2\left(3 \alpha+\beta^{2} \lambda\right) t}{\beta}}}},\left(3 \alpha+\beta^{2} \lambda \neq 0\right.$, i.e., $\left.\gamma \neq 0\right)$

where

$$
\Gamma=\frac{\beta^{2}}{3\left(3 \alpha+\beta^{2} \lambda\right)}-\frac{1}{x_{0}^{2}},\left(3 \alpha+\beta^{2} \lambda \neq 0 \text { or } \gamma \neq 0\right)
$$

The sign of $x(t)$ in Eq. (3.4) is determined as follows: (1) if $x_{0}>0$, then $x>0$ for all $t \geq 0$; (2) if $x_{0}<0$, then $x<0$ for all $t \geq 0$; (3) If $x_{0}=0$, then $x=0$ for all $t \geq 0$.

In Eqs. (3.4) and (3.5), it is assumed that $3 \alpha+\beta^{2} \lambda \neq 0$ or $\gamma \neq 0$. If $3 \alpha+\beta^{2} \lambda=0$ or $\gamma=0$, Eq. (3.3) is rewritten as

$$
\frac{d x}{d t}=-\frac{\beta}{3} x^{3}
$$

The solution to Eq. (3.6) is

$$
x(t)=\frac{ \pm 1}{\sqrt{\Gamma+\frac{2 \beta t}{3}}},\left(3 \alpha+\beta^{2} \lambda=0 \text { or } \gamma=0\right)
$$

where

$$
\Gamma=\frac{1}{x_{0}^{2}}
$$

The sign of $x(t)$ in Eq. (3.7) is similarly determined as before. Comparing the new solution Eqs. (3.4) and (3.7) with the previous solution given in Refs. $[9,10]$, one can find that while both solutions require specific initial conditions Eq. (3.2), the new solution is an explicit solution and is not in a parametric form as in Refs. $[9,10]$.

Figure 1 shows the phase diagram obtained using Eq. (3.4) and its derivative. The displacement $(x)$ and the velocity $(\dot{x})$ of the Duffing-van der Pol system given by Eq. (2.9) are plotted for the case $\alpha=1, \beta=3, \gamma=-4$, and $\lambda=1$. The simulation is shown for a duration of $10 \mathrm{~s}$ and the initial condition is chosen as $\left(x_{0}, \dot{x}_{0}\right)=(3,-15)$, which satisfies Eq. (3.2). As Eq. (3.4) indicates, the displacement converges to $x(t)=1 / \sqrt{\frac{\beta^{2}}{3\left(3 \alpha+\beta^{2} \lambda\right)}}=2$ as time goes to infinity. In Fig. 2 (left), the time history of the displacement of the system is shown by plotting Eq. (3.4). The numerical solution (for these same parameter values) of Eq. (2.9) is computed in the MATLAB environment, using a variable time step ode 45 integrator with a relative error tolerance of $10^{-8}$ and an absolute error tolerance of $10^{-12}$. Figure 2 (right) shows the difference between the analytical solution and the numerical solution. As seen, the difference is small (of the order of $10^{-10}$ ), showing that the numerically obtained solution is consistent with the new analytical result given in Eq. (3.4).

The standard form of the Duffing-van der Pol system has positive $\beta$ and $\lambda=1$ so that Eq. (2.9) can be rewritten as

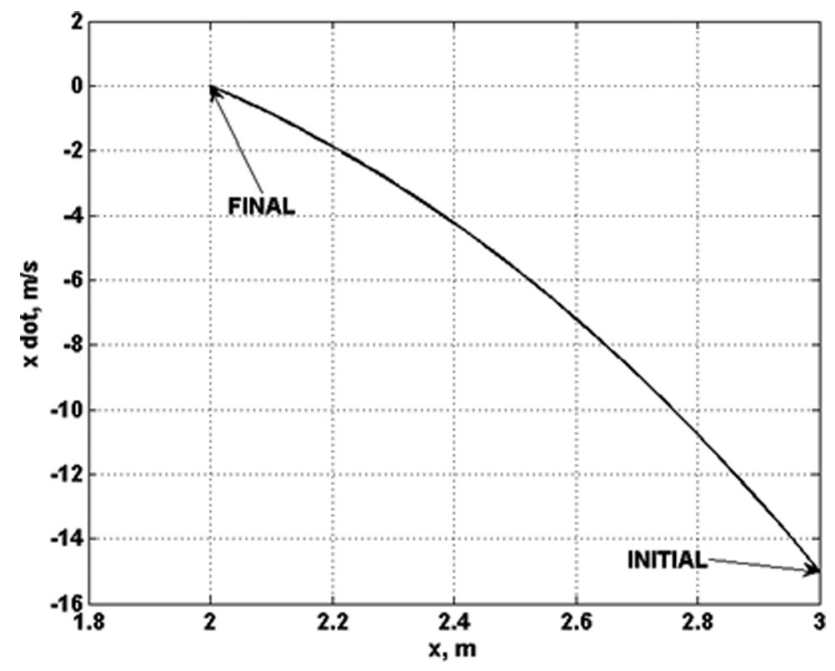

Fig. 1 Phase diagram of the Duffing-van der Pol system Eq. (2.9) with $\alpha=1, \beta=3, \gamma=-4$, and $\lambda=1$ 

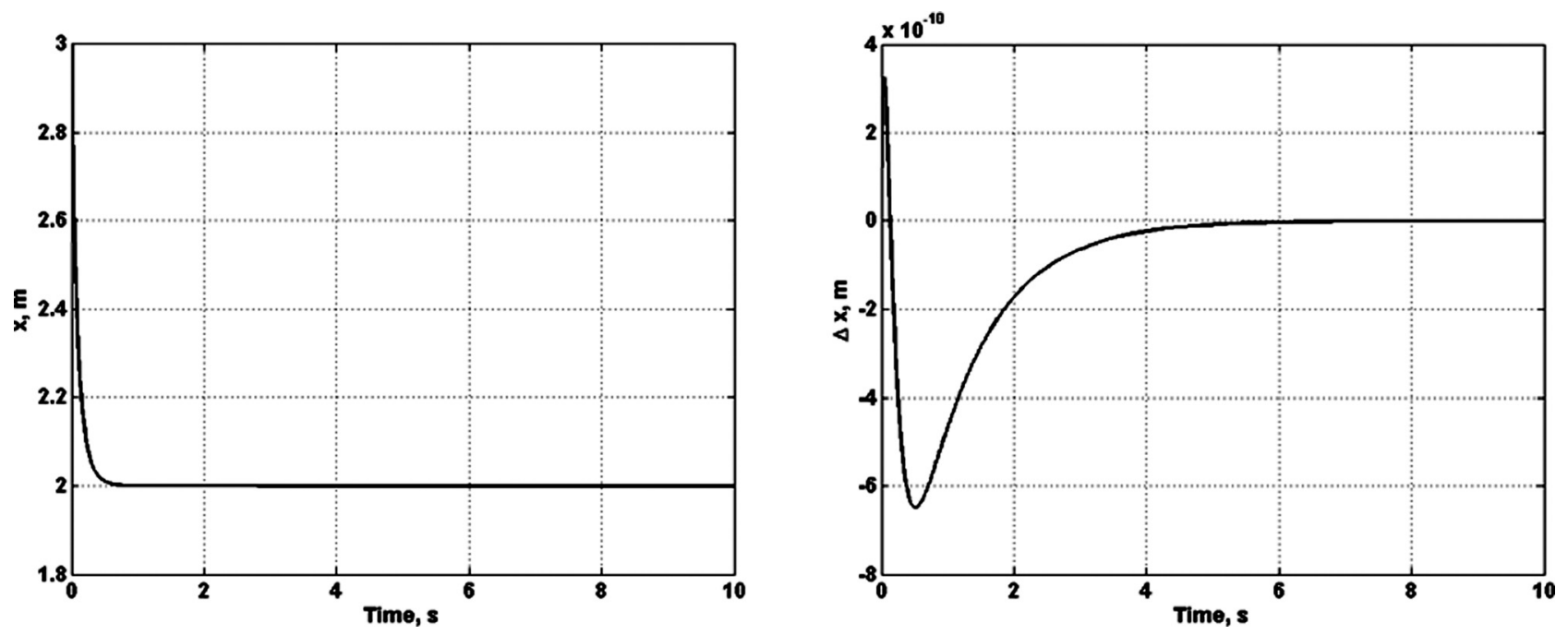

Fig. 2 Time history of the displacement of the system (left) and the difference between the numerical and the new analytical solutions Eq. (3.4) (right)

$$
\ddot{x}+\beta\left(x^{2}-1\right) \dot{x}+\kappa_{1} x+\kappa_{2} x^{3}=0, \quad \beta>0
$$

with

$$
\kappa_{1}=-3 \alpha\left(1+\frac{3 \alpha}{\beta^{2}}\right)=\frac{\beta v-1}{v^{2}}, \quad \text { and } \quad \kappa_{2}=\alpha=-\frac{\beta}{3 v}
$$

In the modeling of most mechanical systems, the coefficient $\kappa_{1}$ is usually positive, signifying a positive "spring" stiffness. Our results go beyond those in Ref. [9] where only the case when $\kappa_{1}$ is negative and $\kappa_{2}=1$ is handled.

Perhaps the most useful characteristic in modeling physical systems by the Duffing-van der Pol system is the self-excited oscillation property that the system can exhibit. Unfortunately, limit cycles do not appear in Eq. (3.9) when the stiffness term $\kappa_{1}>0$ (see the Appendix). This appears to be connected to the fact that the first integrals and the Lagrangians for both the standard van der Pol oscillator and the Duffing-van der Pol oscillator are not known to date.

One can find another solution when $I \neq 0$. From Eq. (2.14), we have the following first-order differential equation:

$$
\frac{d x}{d t}=a_{1} x-a_{2} x^{3}+I e^{-\frac{3 x}{\beta} t}
$$

where

$$
a_{1}=\frac{3 \alpha+\beta^{2} \lambda}{\beta}, \quad a_{2}=\frac{\beta}{3}
$$

Using the transformation

$$
x(t)=u(t) e^{a_{1} t}
$$

Eq. (3.11) becomes

$$
\frac{d u}{d t}=-a_{2} u^{3} e^{2 a_{1} t}+I e^{-\left(\frac{3 \alpha}{\beta}+a_{1}\right) t}
$$

A second transformation

$$
T=\frac{a_{2}}{2 a_{1}} e^{2 a_{1} t}
$$

where we assume $a_{1} \neq 0$, further reduces Eq. (3.14) to the firstorder equation

$$
\frac{d u}{d T}=-u^{3}+I_{1} T^{\eta}
$$

where

$$
I_{1}=\left(\frac{2 a_{1}}{a_{2}}\right)^{\eta} \frac{I}{a_{2}} \quad \text { and } \quad \eta=-\frac{3\left(4 \alpha+\beta^{2} \lambda\right)}{2\left(3 \alpha+\beta^{2} \lambda\right)}
$$

Although simple and elegant, Eq. (3.16) is of the so-called Abel type, and it is difficult to get analytical solutions for it. Closedform solutions can be found when $\eta=-3 / 2$ and $\eta=0$ at which values the equation is variable separable. When $\eta=-3 / 2$, however, the second of Eq. (3.17) would require $\alpha=0$, and from Eq. (2.9), this would mean that we no longer have a Duffing-van der Pol system! Hence we consider only the case when $\eta=0$, i.e., $\alpha=-\left(\beta^{2} \lambda\right) / 4$. Then, Eq. (3.16) gives

$$
\int \frac{d u}{I_{1}-u^{3}}=\int d T
$$

and integration on both sides yields

$$
\frac{1}{6 d^{2}}\left[\ln \frac{d^{2}+d u+u^{2}}{(d-u)^{2}}+2 \sqrt{3} \tan ^{-1}\left(\frac{2 u+d}{\sqrt{3} d}\right)\right]=T+C
$$

where $C$ is an integration constant, and

$$
d=I_{1}^{1 / 3}=\left(\frac{3 I}{\beta}\right)^{1 / 3}
$$

Using the transformations Eqs. (3.13) and (3.15), Eq. (3.19) is described in terms of $x(t)$ and $t$ as

$$
\begin{aligned}
& \frac{1}{6 d^{2}}\left[\ln \left\{\frac{d^{2}+d x e^{-\frac{\beta \lambda}{4} t}+x^{2} e^{-\frac{\beta \lambda}{2} t}}{\left(d-x e^{-\frac{\beta \lambda}{4} t}\right)^{2}}\right\}+2 \sqrt{3} \tan ^{-1}\left(\frac{2 x e^{-\frac{\beta \lambda}{4} t}+d}{\sqrt{3} d}\right)\right] \\
& =\frac{2}{3 \lambda} e^{\frac{\beta \lambda}{2} t}+C
\end{aligned}
$$

where

$$
C=\frac{1}{6 d^{2}}\left[\ln \left\{\frac{d^{2}+d x_{0}+x_{0}^{2}}{\left(d-x_{0}\right)^{2}}\right\}+2 \sqrt{3} \tan ^{-1}\left(\frac{2 x_{0}+d}{\sqrt{3} d}\right)\right]-\frac{2}{3 \lambda}
$$


In brief, Eq. (3.21) gives the closed-form solution to the following Duffing-van der Pol system:

$$
\ddot{x}+\beta\left(x^{2}-\lambda\right) \dot{x}+\frac{3 \beta^{2} \lambda^{2}}{16} x-\frac{\beta^{2} \lambda}{4} x^{3}=0
$$

The solution is valid for all initial conditions.

\section{Conclusions}

In this paper, a new and simple method is introduced for obtaining the first integrals of the Duffing-van der Pol type equation under a wider set of circumstances than obtained hereto. New solutions are also obtained. Comparing with the existing methods such as the Prelle-Singer method [6] and the Lie symmetry method [8], this approach is much simpler and makes it possible to derive new and more general solutions with weaker parametric constraints than hereto known. The simplicity lies in the fact that the Duffing-van der Pol type equation can be transformed into a special form that admits total integrability in certain situations. Although the approach employed herein is simple and easy, a more rigorous and systematic way for arriving at the first integrals and the solutions under more general conditions needs much more work. At present, it remains an open problem. In addition, we still have no first integrals for the standard van der Pol system which has no cubic term, that is, when $\alpha=0$ in Eq. (2.1). As seen here, the cubic term plays an important role in our approach for finding the first integrals and the solutions. In future work, more general cases will be considered and other proper transformations that allow total integrability will be developed.

\section{Appendix}

We show that the Duffing-van der Pol equation in Eq. (3.9) does not have any limit cycle surrounding the origin when $\kappa_{1}>0$, i.e., when $\beta \nu>1$. We replace $t$ by $-t$ in Eq. (3.9) so that we are now investigating what happens as $t \rightarrow-\infty$. The orbits remain the same but their direction is reversed. In particular, any limit cycle $L$ is unchanged in location in the phase portrait. Equation (3.9) then becomes

$$
\ddot{x}+\beta\left(1-x^{2}\right) \dot{x}+\kappa_{1} x+\kappa_{2} x^{3}=0, \quad \beta>0
$$

Denoting

$$
\begin{aligned}
& f(x)=\beta\left(1-x^{2}\right), \quad g(x)=\frac{\beta \nu-1}{\nu^{2}} x-\frac{\beta}{3 \nu} x^{3}, \\
& F(x)=\int_{0}^{x} f(u) d u=\beta x\left(1-x^{2} / 3\right)
\end{aligned}
$$

Eq. (A1) can be rewritten as

$$
\begin{aligned}
& \dot{x}=y-F(x) \\
& \dot{y}=-g(x)
\end{aligned}
$$

The fixed points of this dynamical system are located at $O \equiv(0,0), \quad A \equiv\left(x^{*}, x^{*} / v\right), \quad$ and $\quad B \equiv\left(-x^{*},-x^{*} / v\right)$, where $x^{*}=\sqrt{3\left[1-(\beta \nu)^{-1}\right]}$. Linearization of the above equations about these fixed points reveals that the fixed point at the origin, $O$, is a stable node, while the other two fixed points are saddles. One therefore cannot have a limit cycle around the origin that includes all three fixed points because the sum of the indices of these fixed points would add to -1 ; in a similar fashion, one cannot have a limit cycle around the origin that includes the origin and only one of the saddle points. Thus, the only limit cycle around the origin $O$ that is possible is one that (i) only includes the origin and (ii) cuts the (open) line segment $A O B$ at two points, one on either side of $O$, so that none of the saddle points are included in the cycle. We next show that such a limit cycle is not possible.

Consider an orbit through the point $\left(x_{0}, x_{0} / v\right)$, where $x_{0} \neq 0$ and $x_{0} \neq \pm x^{*}$. Such an orbit of Eq. (A3), which does not start at any of the three fixed points, always satisfies the equation

$$
y(t)=x(t) / \nu
$$

because from Eqs. (A2) and (A3) $\dot{y}(t)=\left(x(t) / \nu^{2}\right)-(\beta x(t) / v)$ $+\left(\beta x(t)^{3} / 3 v\right)=\dot{x}(t) / v$ is satisfied.

As such, the line $y(t)=x(t) / \nu$ represents an orbit in the phase plane, and since two orbits cannot cross, there can be no limit cycle whose orbit includes the origin and cuts the line segment $A B$.

\section{References}

[1] Santilli, R. M., 1978, Foundations of Theoretical Mechanics I: The Inverse Problem in Newtonian Mechanics, Springer-Verlag, New York.

[2] Leitmann, G., 1963, “Some Remarks on Hamilton's Principle," ASME J. Appl. Mech., 30, pp. 623-625.

[3] Udwadia, F. E., Leitmann, G., and Cho, H., 2011, "Some Further Remarks on Hamilton's Principle,” ASME J. Appl. Mech., 78, p. 011014.

[4] Udwadia, F. E., and Cho, H., 2012, "Lagrangians for Damped Linear MultiDegree-of-Freedom Systems," ASME J. Appl. Mech., 80, p. 041023.

[5] Pars, L. A., 1972, A Treatise on Analytical Dynamics, Ox Bow Press, Woodbridge, CT.

[6] Prelle, M., and Singer, M., 1983, "Elementary First Integrals of Differential Equations," Trans. Am. Math. Soc., 279, pp. 215-229.

[7] Duarte, L. G. S., Duarte, S. E. S., da Mota, A. C. P., and Skea, J. E. F., 2001, "Solving the Second-Order Ordinary Differential Equations by Extending the Prelle-Singer Method," J. Phys. A, 34, pp. 3015-3024.

[8] Hydon, P. E., 2000, Symmetry Methods for Differential Equations, Cambridge University Press, New York.

[9] Gao, G., and Feng, Z., 2010, "First Integrals for the Duffing-van der Pol Type Oscillator," Electron. J. Differ. Equations, 19, pp. 1-12.

[10] Feng, Z., Gao, G., and Cui, J., 2011, "Duffing-van der Pol Type Oscillator System and Its First Integrals," Commun. Pure Appl. Anal., 10, pp. 1377-1391. 